\title{
An approach to the limb darkening of irradiated stellar atmospheres *
}

\author{
A. Claret \\ Instituto de Astrofísica de Andalucía, CSIC, Apartado 3004, 18080 Granada, Spain \\ e-mail: claret@iaa.es
}

Received 14 February 2007 / Accepted 9 May 2007

ABSTRACT

\begin{abstract}
Context. The effects of irradiation on stellar limb darkening is still complicated to implement in light curve synthesis codes since it involves a large combination of parameters, including the effective temperatures of the source/irradiated stars, the apparent radius, and the angle of incidence of the infalling radiation field. This makes it too difficult to generalize how irradiation affects the limb darkening coefficients (LDCs).

Aims. The aim of this paper is to present, in a suitable manner to be used directly in light curve synthesis codes, the effects of irradiation on the LDCs of convective atmospheres.

Methods. To investigate the mutual irradiation of close binary systems, we have adopted a modified version of the UMA model atmosphere code with the upper boundary condition changed to take into account the external flux. Numerical improvements are also introduced, concerning the spatial resolution of the code (the number of $\mu$ points is increased from 6 to 18 points) and more modern models of atmospheres are adopted to simulate the hot companions.

Results. We have systematically studied the effects of irradiation on the LDCs of stellar atmospheres and present corrections to the LDCs to account for such effects. These corrections are given as a function of the relative flux for 12 commonly used passbands (Johnson-Cousins $U B V R I J H K$ and Strömgren $u v b y$ ) and can be easily implemented in light curve synthesis codes. A qualitative comparison with the few empirical measurements of LDCs indicates that the theoretical corrections give LDCs in better agreement with observations, particularly for interacting binary systems.
\end{abstract}

Key words. stars: binaries: close - stars: binaries: eclipsing

\section{Introduction}

One of the most important challenges to our ability to model the light curves of eclipsing binaries is to take into account proximity effects. These effects include distortions by rotation and tidal contributions and mutual irradiation by the binary components. These proximity effects alter the physics of the problem, substantially increasing the degree of complexity of the theory involved. The distortions by rotation and tides can be described through the apsidal-motion constants (Kopal 1959). However, some simplifications are assumed in order to derive such a relationship. It is often assumed that a star on an eccentric orbit readjusts its shape instantaneously during its revolution around the centre of mass of the system (this is only valid when the orbital period is much larger than the periods of the low-order modes of oscillation). More recent investigations have considered the effects of the stellar compressibility and mainly the effects of resonances (Papaloizou \& Pringle 1980; Quataert et al. 1996, Smeyers \& Willems 2001; Willems \& Claret 2002). As a result of these improvements, the dynamical tides were systematically taken into account to determine the contribution of the tidal distortion to the predicted apsidal motion rate, which can be compared with observational data (see Claret \& Willems 2002).

The study of rapidly rotating or tidally distorted stars requires knowledge of how the flux is distributed over the stellar surface. The brightness distribution depends on the gravity

\footnotetext{
* Additional data are available upon request.
}

darkening exponent $\beta_{1}$. The first investigation of gravity darkening was by von Zeipel (1924), who determined that the flux is directly proportional to the local gravity for stars with radiative envelopes: $T_{\text {eff }}^{4} \propto g^{\beta_{1}}$ with $\beta_{1}=1$. Investigations for convective envelopes were carried out about 40 years later by Lucy (1967). It was found that $\beta_{1}$ is of the order of 0.32 , a very different value from the radiative case. Since then, the values of 1.0 and 0.32 have been used by light curve modellers until the work by Claret (1998, 2004a), who used a new computational procedure - the triangle strategy - and was able to derive $\beta_{1}$ as a function of mass, chemical composition and age. The old values of $\beta_{1}$ for convective and radiative envelopes are superseded by those recent calculations and a smooth transition is achieved between the radiative and convective energy transport mechanisms.

The situation of the mutual irradiation of the components of a close binary and, in particular, the computation of limb darkening coefficients (LDCs), is much more complicated. Mutual irradiation in a close binary star may alter several characteristics of the system: the effective temperature and the respective position in the HR diagram are changed, mainly in the case of the secondary stars (Claret \& Giménez 1992) and the limb darkening may be highly altered (Claret 2004b). Concerning only limb darkening, the main complication comes from the variety of combinations of irradiation parameters such as the relative distance between the components, the effective temperatures, the direction of the irradiating beam, etc. This makes it difficult to generalize the effects of irradiation on the LDCs. In this paper we try to systematically account for these effects in order to 
make available to light curve modellers theoretical LDCs corrected for the effect of irradiation. These corrections are given as a function of the relative flux for 12 commonly used photometric passbands.

\section{Numerical method}

Irradiated models were generated by changing the upper boundary condition adopted by the model atmosphere UMA (Gustafsson et al. 1975; Nordlund \& Vaz 1990; Claret \& Giménez 1992). The specific intensity at the optical depth $\tau=0$ is changed due to the external flux as:

$I\left(0, \mu_{j}\right)=2 u_{1, j} \mathrm{e}^{\frac{-\tau_{1}}{\mu_{j}}}+S_{1}\left(1-\mathrm{e}^{\frac{-\tau_{1}}{\mu_{j}}}\right)^{2}+i_{\text {ext }} \mathrm{e}^{\frac{-2 \tau_{1}}{\mu_{j}}} \delta_{i j} h^{-1}\left(\mu_{i}\right)$

where

$u\left(\tau_{k}, \mu_{j}\right)=\frac{1}{2}\left[I\left(\tau_{k}, \mu_{j}\right)+I\left(\tau_{k},-\mu_{j}\right)\right]$

and the external radiation field is given by

$I(-\mu, \eta)=i_{\mathrm{ext}} \delta\left(\mu-\mu_{i}\right) \delta\left(\eta-\eta_{i}\right)$

In the above equations $S_{1}$ is the source function, $i_{\mathrm{ext}}$ is the constant which characterizes the external radiation field, the symbol $\delta_{i j}$ denotes the Kronecker function while $h\left(\mu_{j}\right)$ are the Gaussian weights, $\mu_{i}$ is the cosine of the incidence angle and $\eta_{i}$ is the azimuthal angle. Note that due to the discretization of the emergence angles $\mu$, we can only simulate irradiated atmospheres in discrete and pre-selected directions (in the present case, four). The $\mu$ vary from 0.07 (grazing angle) up to 0.93 (almost perpendicular). Another limitation concerns the grid of stellar models by Kurucz (2000) which characterizes the external flux. As the wavelength values used in UMA are different to those used by ATLAS we have to carry out interpolations to obtain suitable intensities to be used in the UMA code. Such interpolations imply a net loss of flux, mainly in the ultraviolet. As a consequence, the resulting effective temperatures of the ATLAS models are somewhat different than tabulated. This point is very important in the computation of the bolometric albedos. The introduction of the more recent grids by Kurucz is an improvement since previous investigations on irradiation using UMA adopted the old stellar atmosphere models given by Kurucz (1979). To obtain the irradiated models we have started with trial models with arbitrary albedos. Their pressure-temperature relationships are then compared with that of the non-irradiated model. The process is iterated until the irradiated and original non-irradiated model present the same entropy at the bottom of the atmosphere.

In a previous work on the effects of external radiation field on the limb darkening (Claret 2004b) we emphasized the role of the numerical method to derive the LDCs. The results are presented for very specific conditions of irradiation. In order to generalize the effects of irradiation on the LDCs we have generated a large series of models under several conditions of irradiation: relative distances, effective temperatures of the irradiating and irradiated stars, etc. The results are presented in the most commonly used photometric systems: Strömgren $u v b y$ and Johnson-Cousins $U B V R I J H K$. The specific intensity for a given photometric band $a$ is given by

$I_{a}(\mu)=\frac{\int_{\lambda_{1}}^{\lambda_{2}} I(\lambda, \mu) S(\lambda) \mathrm{d} \lambda}{\int_{\lambda_{1}}^{\lambda_{2}} S(\lambda) \mathrm{d} \lambda}$ where $I(\lambda, \mu)$ is the monochromatic specific intensity and $S(\lambda)$ is the response function (the terrestrial atmospheric transmission, filter transmission curves, detector sensitivity and double reflection from the aluminum coated mirror). More details on the response function can be found in our previous papers. The most commonly used limb darkening laws are the linear, the quadratic, the square root and the logarithmic. As almost all observations of limb darkening (see Sect. 3) make use of the linear law and also for clarity, we concentrate our calculations on this approximation. However, there are some reliable applications/derivations of non-linear LDC. The high quality HST observations of the transits of an extrasolar planet located in the system HD 209458 and non-linear LDCs were used to refine the physical properties of HD 209458b (Knutson et al. 2007). Concerning eclipsing binaries, Southworth, Bruntt $\&$ Buzasi (2007) obtained around 30000 datapoints for $\beta$ Aur using the WIRE satellite. The quality and the high precision of such a set of data allowed them to infer non-linear coefficients since they improve the quality of the fit. Given that the derivation of non-linear LDCs are increasingly important and frequent, we also analysed them here. The cases of non-linear laws can be obtained directly from the author, upon request.

The linear law is

$\frac{I(\mu)}{I(1)}=1-u(1-\mu)$

where all symbols have their usual meaning. If more accurate light curve modelling is required we refer to a new limb darkening law, $\frac{I(\mu)}{I(1)}=1-\sum_{k=1}^{4} a_{k}\left(1-\mu^{\frac{k}{2}}\right)$, introduced in Claret (2000). In this case a more careful treatment of the effects of irradiation should be carried out for each specific system.

Limb darkening investigations need a good spatial resolution, and the usual angle resolution used in UMA was increased to $18 \mu$ points instead of only 6 adopted previously. This improvement is shown to be crucial to detect and quantify the limb brightening of illuminated atmospheres (when present) and, of course, in the discussion of the best method to derive the LDCs (least-squares, LSM, or flux conservation method, FCM). For more detail see Claret (2004b).

\section{Corrections to the limb darkening of irradiated atmospheres}

The irradiated models are in principle very different from the non-irradiated ones (with same effective temperature and $\log g$ ) not only concerning the intensity distribution on the disk but also with respect to their resulting spectra. Under the determined conditions of irradiation the intensity distribution is so altered that limb brightening is detected instead of the usual limb darkening. Such a phenomenon has also been detected in standard model atmospheres, as shown by Wade \& Ruciński (1985) and Claret (2000) for the ATLAS and PHOENIX codes. That behaviour is probably connected with unrealistic temperature inversions. As noted in the previous section, a better sampling of the emergence angle $\mu$ is necessary and we have modified our UMA version to include 12 new points which are important in the study of the resulting intensity distribution, mainly near the limb (Fig. 1 in Claret 2004b). Note that is possible to detect limb brightening with these extra $\mu$ points, but when using the usual $6 \mu$ points it is only possible to detect a more uniform distribution and not a limb brightening. Also, the intensity distribution of the non-irradiated model with the same effective temperature as the irradiated model is different from the irradiated one. 

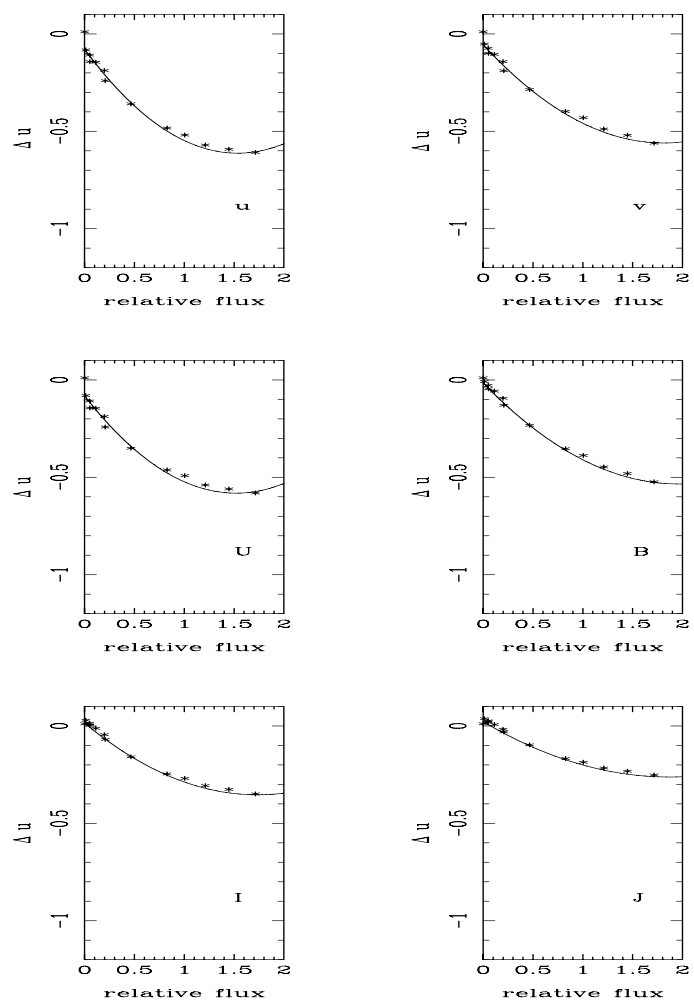
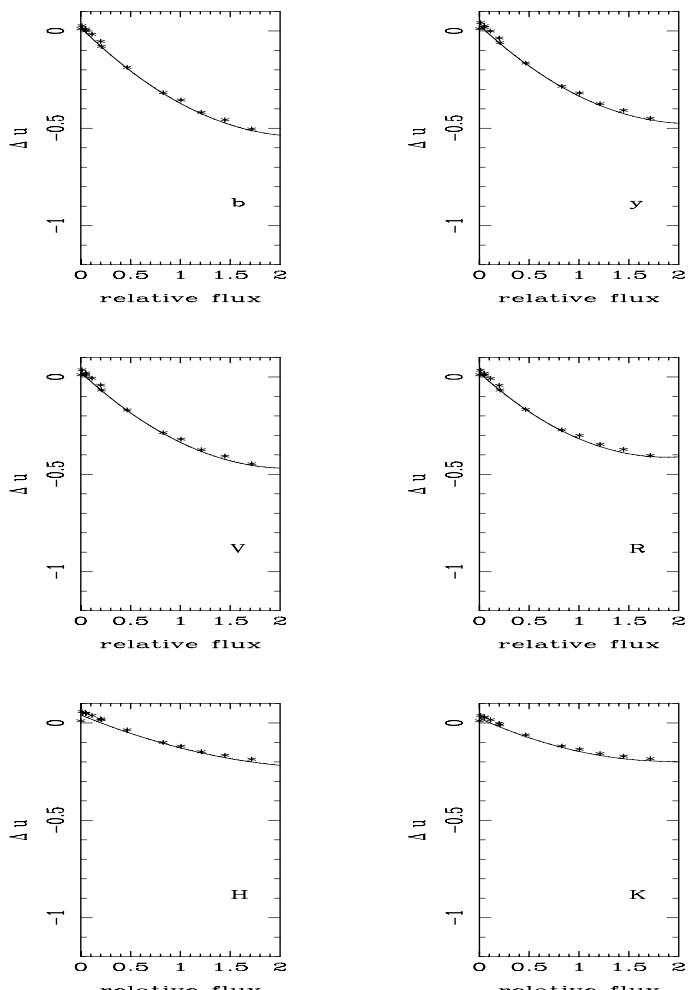

Fig. 1. Corrections to the linear LDC as a function of the relative flux. The letters inside the boxes denote the passbands. Coefficient $u$. $T_{\text {eff }}=4000 \mathrm{~K}$.

The more the external flux increases the more the irradiated model is affected. However, our present model of irradiation still presents limitations and includes some approximations, such as the introduction of the external radiation field itself. For example, the auxiliary function $u_{k, j}$ which appears in Eq. (2) (and its counterpart $v_{k, j}$ ) is expanded in Taylor series only up to the second order. Another source of uncertainty is the circulation currents, which are not considered here (Kirbiyik 1982), and the plane-parallel approach used in UMA instead of adopting a more elaborate spherical geometry. Concerning this last point, the intensity distribution for models with spherical symmetry is very different from otherwise similar plane-parallel models (see for example, Orosz \& Hauschildt 2000; and more recently Claret \& Hauschildt 2003). Due to these limitations, and others listed by Claret (2004b), we have restricted our calculations to situations where the relative external flux is within a factor of about 2 of the flux due to the star under consideration. The relative external flux is given by $F_{r}^{\mathrm{rel}} \equiv F_{r} / \sigma T_{\mathrm{eff}}^{4}=T^{* 4} r^{2} v / T_{\mathrm{eff}}^{4}$, where $T^{*}$ is the effective temperature of the irradiating star, $r$ the apparent radius, $v$ is the cosine of the incidence angle and $T_{\text {eff }}$ is the effective temperature without irradiation. We have computed irradiated models with $T^{*}$ varying from $5000 \mathrm{~K}$ up to $13000 \mathrm{~K}, r$ varying from 0.1 up to $0.4, T_{\text {eff }}$ varying from $3500 \mathrm{~K}$ up to $6500 \mathrm{~K}$, and for four values of $v(0.07,0.33,0.67,0.93)$. Another restriction we have imposed concerns the ratio of the effective temperature of the irradiating star, $T^{*}$, and the effective temperature without irradiation, $T_{\text {eff }}$. Due to the lack of wavelengths in the ultraviolet in the UMA code (see above) this spectral region cannot be conveniently treated as the infalling flux is not correctly taken into account. Moreover, the resulting irradiated model is highly perturbed in this spectral region if the ratio $T^{*} / T_{\text {eff }}$ is large. We restrict this ratio to values smaller than 2 .

To use LDCs of non-irradiated models to simulate the irradiated ones is not the best procedure. As we have seen the intensity distribution of an irradiated model is very different to that of a standard one (without external flux). However, in practice it would be interesting to directly use the available tabulations of standard LDCs and correct them for irradiation, at least as a first approach. This was done here by plotting the differences between the LDC of irradiated models, and those from non-irradiated models but with the same effective temperatures, as a function of the relative flux for the 12 passbands: uvbyUBVRIJHK. The results can be seen in Fig. 1 for the case of $T_{\text {eff }}=4000 \mathrm{~K}$ and Fig. 2 for the case of $T_{\text {eff }}=6000 \mathrm{~K}$. The corrected component of the LDCs is given, for each filter $k$, by:

$L D C_{\text {irra }}^{k}=L D C_{\text {stan }}^{k}+\Delta L D C^{k}$

where $\Delta L D C^{k}=a_{k} F_{r}^{\mathrm{rel} 2}+b_{k} F_{r}^{\mathrm{rel}}$. A least-square fitting method was used to determine the LDCs, which are given in Table 1. As a boundary condition, we have imposed that the fitted curve passes through zero. The average $\sigma$ of the fittings are of the order of $10^{-3}$ though they are larger for lower effective temperatures, for example $T_{\text {eff }}=3500 \mathrm{~K}$. Note that the term $L D C_{\text {stan }}^{k i}$ refers to the LDCs of a standard (non-irradiated) model with effective temperature corrected for irradiation following $\sigma T_{h}^{4}=$ $\sigma T_{\text {eff }}^{4}+A F_{r}$, where $A$ is the bolometric albedo. The corrections should be applied through an iterative procedure in light curve synthesis codes, using as trial parameters the albedos, relative distances and effective temperatures $\left(A, r, T_{\text {eff }}, T_{h}, T^{*}\right)$. Changes in the incidence angle do not change the general trend of Figs. 1 and 2 but increase somewhat the corresponding scattering. If more accuracy is necessary, as required by some light curve codes, we can also supply, in addition to the corrections given in Eq. (6), the resulting specific intensities for each irradiation configuration. This makes it possible to eliminate the intrinsic uncertainties in Eq. (6) and also in the direct use of the LDCs.

Note that the relative flux is diluted by the geometric factors $r^{2}$ and $v$. For a typical Algol-type system we have $F_{r}^{\text {rel }} \approx 1$ 

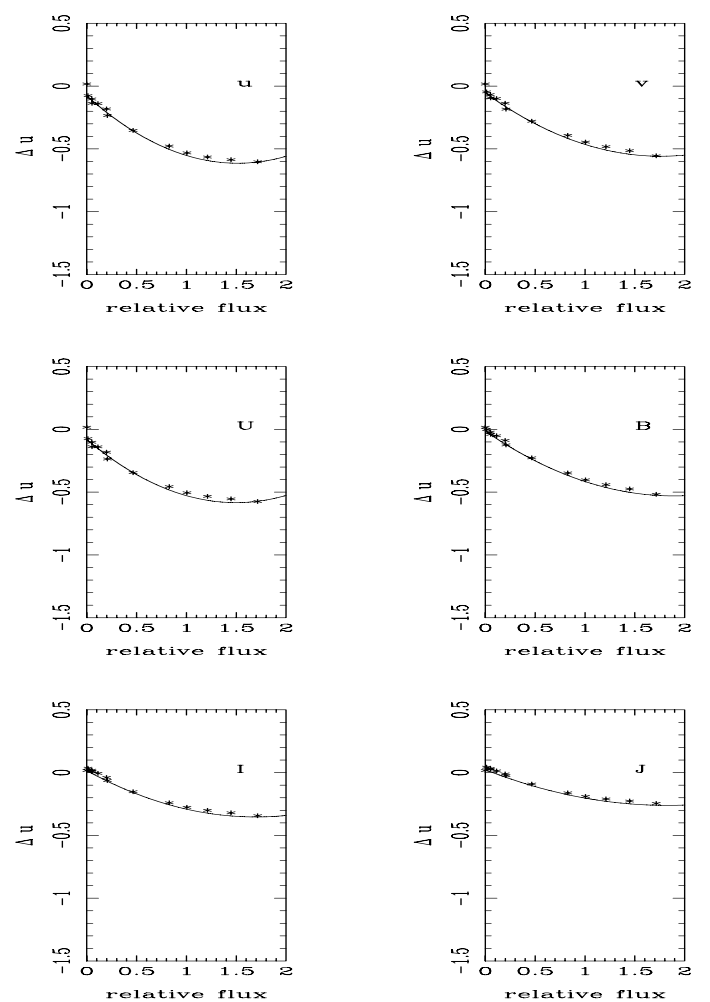
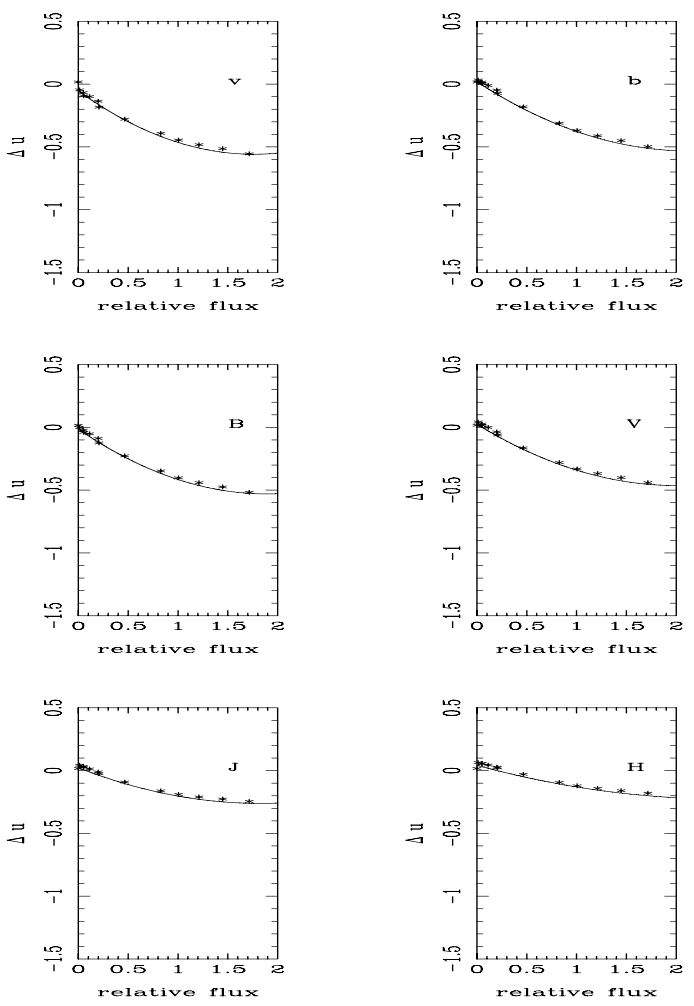
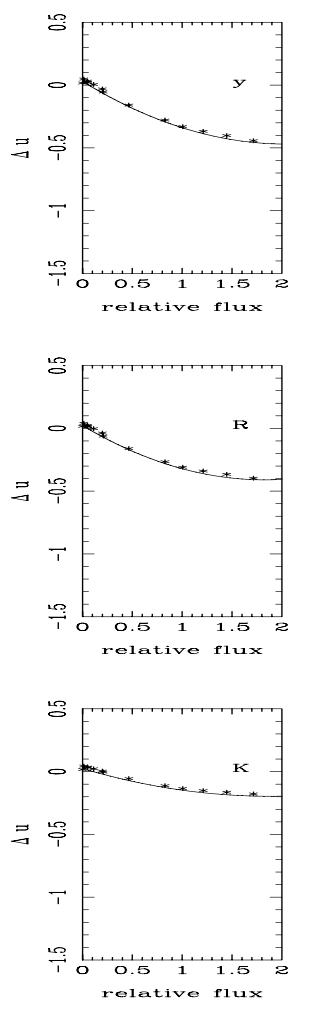

Fig. 2. The same as in Fig. 1 but for $T_{\text {eff }}=6000 \mathrm{~K}$.

Table 1. Coefficients for correction of irradiated LDC.

\begin{tabular}{|c|c|c|c|c|c|c|c|c|c|c|c|c|c|}
\hline & $T_{\text {eff }}$ & $u$ & $\bar{v}$ & $b$ & $y$ & $\overline{\bar{U}}$ & $\overline{\bar{B}}$ & $\overline{\bar{V}}$ & $\overline{\bar{R}}$ & $\bar{I}$ & $\bar{J}$ & $\overline{\bar{H}}$ & $\overline{\bar{K}}$ \\
\hline Linear $\mathrm{u} \mathrm{a}_{k}$ & 3500 . & 0.73 & 0.48 & 0.79 & 0.67 & 0.74 & 0.63 & 0.61 & 0.42 & 0.38 & 0.06 & -0.11 & -0.06 \\
\hline Linear $\mathrm{u} \mathrm{b}_{k}$ & 3500 . & -2.11 & -1.64 & -2.09 & -1.79 & -2.09 & -1.82 & -1.67 & -1.30 & -1.14 & -0.46 & -0.09 & -0.13 \\
\hline Linear $\mathrm{u} \mathrm{a}_{k}$ & 4000 . & 0.47 & 0.42 & 0.50 & 0.39 & 0.49 & 0.45 & 0.37 & 0.28 & 0.27 & 0.15 & 0.00 & 0.04 \\
\hline Linear $\mathrm{u} \mathrm{b}_{k}$ & 4000 . & -1.41 & -1.30 & -1.36 & -1.12 & -1.42 & -1.29 & -1.08 & -0.88 & -0.81 & -0.53 & -0.18 & -0.24 \\
\hline Linear $\mathrm{u} \mathrm{a}_{k}$ & 4500 . & 0.36 & 0.35 & 0.29 & 0.22 & 0.37 & 0.32 & 0.23 & 0.18 & 0.18 & 0.12 & 0.01 & 0.04 \\
\hline Linear $\mathrm{u} \mathrm{b}_{k}$ & 4500 . & -1.07 & -1.03 & -0.87 & -0.71 & -1.08 & -0.93 & -0.73 & -0.62 & -0.58 & -0.40 & -0.15 & -0.21 \\
\hline Linear $\mathrm{u} \mathrm{a}_{k}$ & 5000. & 0.47 & 0.45 & 0.28 & 0.21 & 0.49 & 0.35 & 0.23 & 0.20 & 0.21 & 0.12 & 0.00 & 0.04 \\
\hline Linear $\mathrm{u} \mathrm{b}_{k}$ & 5000 . & -1.19 & -1.11 & -0.78 & -0.65 & -1.21 & -0.92 & -0.68 & -0.60 & -0.58 & -0.37 & -0.13 & -0.19 \\
\hline Linear $\mathrm{u} \mathrm{a}_{k}$ & 5500. & 0.40 & 0.35 & 0.16 & 0.13 & 0.42 & 0.25 & 0.14 & 0.14 & 0.15 & 0.08 & -0.01 & 0.03 \\
\hline Linear $\mathrm{u} \mathrm{b}_{k}$ & 5500. & -1.06 & -0.92 & -0.59 & -0.50 & -1.06 & -0.74 & -0.52 & -0.49 & -0.48 & -0.30 & -0.10 & -0.17 \\
\hline Linear $\mathrm{u} \mathrm{a}_{k}$ & 6000. & 0.34 & 0.23 & 0.10 & 0.08 & 0.33 & 0.16 & 0.09 & 0.10 & 0.11 & 0.05 & -0.01 & 0.03 \\
\hline Linear $\mathrm{u} \mathrm{b}_{k}$ & 6000. & -0.91 & -0.72 & -0.47 & -0.41 & -0.89 & -0.58 & -0.42 & -0.41 & -0.39 & -0.25 & -0.10 & -0.17 \\
\hline Linear $\mathrm{u} \mathrm{a}_{k}$ & 6500 . & 0.31 & 0.16 & 0.05 & 0.04 & 0.28 & 0.11 & 0.05 & 0.07 & 0.08 & 0.04 & -0.02 & 0.03 \\
\hline Linear $\mathrm{u} \mathrm{b}_{k}$ & 6500 . & -0.85 & -0.58 & -0.39 & -0.34 & -0.78 & -0.48 & -0.35 & -0.36 & -0.33 & -0.22 & -0.10 & -0.17 \\
\hline
\end{tabular}

and for a detached system with similar main-sequence components $F_{r}^{\mathrm{rel}} \approx 0.03$. In the last case, the predicted corrections are not very large but still significant. The magnitude of the correction depends on the passband, being smaller for larger effective wavelengths, as expected, since the models are less perturbed in this spectral region. For longer wavelengths the behaviour of the corrections is almost linear, particularly for the hotter models.

An important feature which is apparent in the above figures is that the corrections are systematically negative and the resulting LDCs are smaller than their non-irradiated counterparts. Is it possible to contrast this result with some observational evidence? The observation of LDCs using double-lined eclipsing binaries is not a simple task (Popper 1984) and the derived coefficients are still not reliable enough to permit a strict comparison. Despite this, Twigg \& Rafert (1980) used the Wilson-Devinney code to obtain LDCs (linear approximation) for several detached, semi-detached and contact systems. Whilst the theoretical LDCs they used to compare with the inferred values are based on very old atmosphere models, they were able to conclude that the detached systems present smaller discrepancies while systems with larger proximity effects gave larger discrepancies. This may indicate (qualitatively speaking) that the discrepancies are larger for systems showing stronger irradiation effects. More recently, works by several authors (see for example Lacy and collaborators in the reference list; Bruntt et al. 2006; Southworth et al. 2004, 2005) have measured linear LDCs from the light curves of detached eclipsing systems. It is well known that the linear approximation is not a good description of the intensity distribution, but these are the few cases where observed LDCs are available. Therefore, we should consider such results with caution. If we compare these measured LDCs with those from standard calculations (without irradiation), for example those by Claret (2000), we find that the measured values are systematically smaller that theoretically predicted. This seems to favour the Flux Conservation Method (FCM) which produces smaller LDCs than those obtained by 
using Least-Squares Method (LSM). However, as discussed by Díaz-Cordovés et al. (1995) and Claret (2000), FCM presents severe problems, including giving $\sigma$ values that are 2 mag larger than those obtained assuming LSM. Another problem is related to the nature of the FCM itself since for non-linear laws extra conditions (often arbitrary) must be introduced. Other numerical methods used to derive LDCs which give more weight to the limb also gives smaller LDCs that those produced by the LSM. However, these methods also present problems when investigating the effects of external irradiation. Here we interpret the mentioned disagreements as due to irradiation effects, at least partially.

Perhaps the main observational support for the present model of irradiation comes from interacting binary systems, such as the case of EF Eri (a magnetic cataclysmic variable, see Harrison et al. 2003). For this binary the effects of irradiation on the LDCs seem to be more conspicuous than the case of traditional detached systems. The effective temperature of the irradiated side is almost $60 \%$ larger than the non-irradiated one. These authors find significant changes due to irradiation in the LDCs. On the other hand, Hilditch et al. (1996) analysed three systems containing a hot subdwarf and a low mass/luminosity star (HW Vir, AA Dor and KV Vel) and found it necessary to decrease the standard values of the LDCs for the secondary components in order to match the respective light curves.

Considering the above comparisons, we think that the present model of irradiation, though still simple, is capable of explaining the systematic differences between theoretical and measured LDCs, at least partially. The results shown in Figs. 1 and 2 and Table 1 should be taken with caution, however. They should be considered only as a qualitative description and also as only a guide to help to interpret the observed LDCs of double-lined eclipsing binaries.

\section{Final remarks}

Given the approximations adopted in the irradiation model and the limitations mentioned above, we can consider the results shown in Figs. 1 and 2 as significant. Of course, only a detailed treatment of mutual irradiation, such as that performed in Claret (2004b), can take into account more correctly the effects of irradiation on limb darkening. The use of Eq. (6) is therefore not a substitute for a more complete analysis of the irradiation problem. The present results, though more simple, are useful since they permit the evaluation of the influence of irradiation on LDCs in a quick manner and so can be easily incorporated in light curve synthesis codes. An additional advantage of Eq. (6) is that there is no dependence on the specific conditions of irradiation, i.e., it does not depend directly on the spectral characteristics of the irradiating and irradiated stars, etc. Such generalization was possible by using the relative flux $F_{r}^{\text {rel }}$ (recall that in a more refined treatment the LDCs of irradiated atmospheres depend specifically on the refered characteristics).

We plan to investigate the effects of irradiation on the LDC by adopting models with spherical geometry. It is expected that the effects will be less dramatic near the limb for the same conditions of irradiation, as in the case of the plane-parallel approximation.

Acknowledgements. I thank the anonymous referee for comments and generous suggestions that improved the paper. I am in debt to J. Southworth for his comments and suggestions. The Spanish MEC (AYA2006-06375) is gratefully acknowledged for its support during this work.

\section{References}

Bruntt, H., Southworth, J., Torres, G. et al. 2006, A\&A, 456, 651

Claret, A. 1998, A\&AS, 131, 395

Claret, A. 2004a, A\&A, 424, 919

Claret, A. 2004b, A\&A, 422, 665

Claret, A., \& Giménez, A. 1992, A\&A, 256, 572

Claret, A., \& Willems, B. 2002, A\&A, 388, 518

Díaz-Cordovés, J., Claret, A., \& Giménez, A. 1995, A\&AS, 110, 329

Gustafsson, B., Bell, R. A., Eriksson, K., \& Nordlund, A.. 1975, A\&A, 42, 407

Harrison, T. E., Howell, S. B., Huber, M. E. et al. 2003, AJ, 1252609

Hilditch, R. W., Harries, T. J., \& Hill, G. 1996, MNRAS, 279, 1380

Kirbiyik, K. 1982, MNRAS, 200, 907

Kopal, Z. 1959, Close binary systems, Chapman and Hall, London

Knutson, H. A., Charbonneau, D., \& Noyes, R. W. 2007, ApJ, 655, 564

Kurucz, R. L. 1979, ApJS, 40,

Kurucz, R. L. 2000, private communication

Lacy, C. H. S., Torres, G., Claret, A., \& Sabby, J. A. 2000a, AJ, 120, 3226

Lacy, C. H. S., Torres, G., Claret, A. et al. 2000b, AJ, 119, 1389

Lacy, C. H. S., Torres, G., Claret, A., \& Sabby, J. A. 2002, AJ, 123, 1013

Lacy, C. H. S., Vaz, L. P. R., Claret, A., \& Sabby, J. A. 2003, AJ, 128, 1324

Lacy, C. H. S., Torres, G., Claret, A. et al. 2004a, AJ, 128, 3005

Lacy, C. H. S., Torres, G., Claret, A., \& Sabby, J. A. 2004b, AJ, 128, 1840

Lacy, C. H. S., Torres, G., Claret, A., \& Sabby, J. A. 2004c, AJ, 128, 1340

Lacy, C. H. S., Torres, G., Claret, A., \& Vaz, L. P. R. 2005, AJ, 130, 2838

Lucy, L. B. 1967, Z. Astrophys., 65, 89

Nordlund, A., \& Vaz, L. P. R. 1990, A\&A, 228, 231

Orosz, J. A., \& Hauschildt, P. H. 2003, A\&A, 364, 265

Papaloizou J., Pringle J. E. 1980, MNRAS, 193, 603

Popper, D. M. 1984, AJ, 89, 132

Quataert E. J, Kumar P., \& Ao C. O. 1996, ApJ, 463, 284

Smeyers, P., \& Willems, B. 2001, A\&A, 373, 173

Southworth, J., Bruntt, H., \& Buzasi, D. L. 2007, A\&A, 467, 1215

Southworth, J., Maxted, P. F. L., \& Smalley, B. 2004, MNRAS, 351, 1277

Southworth, J. Smalley, B., Maxted, P. F. L., Claret, A., \& Etzel, P. B. 2005 , MNRAS, 363, 529

Twigg, L. W., Rafert, J. B. 1980, MNRAS, 193, 775

von Zeipel, H. 1924, MNRAS, 84, 665

Wade, R. A., \& Ruciński, S. M. 1985, A\&AS, 60, 471

Willems, B., \& Claret, A. 2002, A\&A, 382, 1009 